\section{Josef Roos}

Die Integrative Onkologie erfreut sich zunehmender Aufmerksamkeit. Begründet wird das Interesse vom wachsenden Patientenwunsch, Methoden der Komplementärmedizin in die onkologische Behandlung aufzunehmen.

Das diesjährige Aeskulap-Forum versuchte, die Herausforderung «Integrative Onkologie» im Sinne einer patientenbezogenen Medizin anzunehmen, die klassische und komplementärmedizinisch basierte Verfahren zusammenführt. Vor allem in der Supportivtherapie gelingt dies zunehmend, da Wissenschaftlichkeit und Empirie der angewandten Methoden hier ineinanderfliessen. Schliesslich widmete sich ein Teil der Vorträge auch ergänzenden Ansätzen in der Tumortherapie.

Das Forum zeigte konkrete Ansätze auf, wie im Alltag bewährte erfahrungsmedizinische Ansätze ohne Widerspruch $\mathrm{zu}$ konventioneller Therapie wissenschaftsfundiert und praxisnah miteinander verbunden werden können.

Für alle Beiträge gelang es, hochkarätige Spezialisten aus dem Bereich der integrativen Medizin zu gewinnen. In den folgenden Abschnitten wird der Inhalt der Referate wiedergegeben.

\section{Prof. Dr. med. Thomas Cerny:}

"Integrative Medizin -

Herausforderung für die Onkologie»

Seit Jahren steht die medizinische Onkologie an der Front der biomedizinischen und pharmazeutischen Forschung, und die diagnostischen und therapeutischen Optionen sowie auch deren Ergebnisse haben sich deutlich verbessert. Personalisierte oder individualisierte zielgerichtete

\title{
Fortbildung
}

Schweiz Z Ganzheitsmed 2012;24:350-354

DOI: $10.1159 / 000345215$

\section{Aeskulap-Forum - ein Rückblick}

\section{«Integrative Onkologie - komplementärmedizinische Ansätze in Verbindung mit klassischer Therapie»}

Die Aeskulap-Klinik führt jedes Jahr ein interdisziplinäres Symposium für Ärztinnen und Ärzte durch. Am 4. Aeskulap-Forum beschäftigten sich die vielen Anwesenden mit der Thematik «Integrative Onkologie - komplementärmedizinische Ansätze in Verbindung mit klassischer Therapie». 10 Referenten aus Deutschland und der Schweiz garantierten für eine hochkarätige Veranstaltung und beste Stimmung.

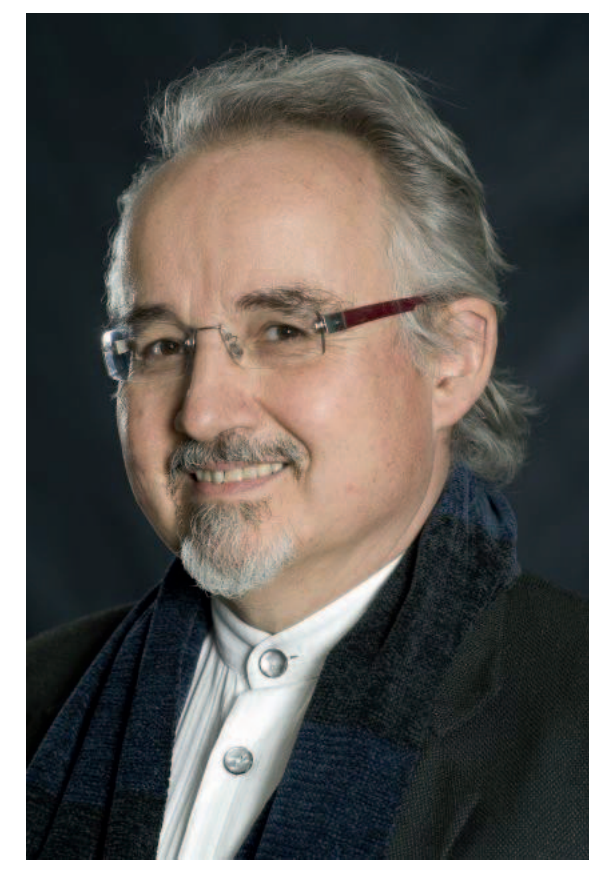

Abb. 1. Prof. Dr. med. Thomas Cerny.

Therapie sind die entsprechenden Schlagworte. Gleichzeitig wird das Durchschnittsalter in der westlichen Welt immer höher, und die Lebenserwartung hat alleine in den letzten 20 Jahren um ca. 5 Jahre in Europa zugenommen. Da Krebs eine altersassoziierte Erkrankung ist, sehen wir immer mehr Patienten mit Malignomen; gleichzeitig können wir die Patienten besser behandeln, sodass sie häufiger geheilt werden und immer länger überleben können. Weltweit gesehen ist Krebs bereits heute die häufigste Todesursache, und die Todesfälle werden sich bis zum Jahr 2030 mehr als verdoppeln. Somit sind auch Früherkennung und Prävention neben einer bestmöglichen Behandlung und Betreuung entscheidende Herausforderungen unserer Zeit. Die Ressourcen sind beschränkt, und dies bezieht sich nicht nur auf die Kosten, sondern genauso auf den absehbaren Mangel an Fachpersonal und Infrastruktur.

Dies alles spielt sich $a b$ in einer sich zunehmend demokratisierenden Welt, die sich im Westen individualistisch-pluralistisch entwickelt hat. So sind die Patientenkompetenz, die Autonomie und das Empowerment Teil eines modernen Medizinverständnisses und rufen nach ganzheitlichen integrativen Therapiekonzepten auch in Zentrumsspitälern. In der Onkologie sind mehr als die Hälfte der Patienten im Verlauf ihrer Krebserkrankung an komplementärmedizinischen Verfahren interessiert und wenden sie auch an. Die Herausforderung einer Integrativen Onkologie an einem Onkologiezentrum liegt vor allem im Bereich einer ausgewogenen Information für die Patienten sowie einer kritischen systematischen und prospektiven Evaluation der benannten Therapieindikationen, der Ergebnisse und der möglichen Kontraindikationen und Gefahren häufig
(๑) 2012 S. Karger GmbH, Freiburg
Dr. Josef Roos

Medienverantwortlicher Aeskulap-Klinik

Gersauerstrasse 8, 6440 Brunnen, Schweiz

josef.roos@aeskulap.com 
nachgefragter komplementärmedizinischer Behandlungen. Im Kontext mit bereits gut etablierten psychoonkologischen, musiktherapeutischen, palliativonkologischen und rehabilitativen Angeboten sowie einer ausgebauten klinischen Forschungsstruktur und -kultur im Bereich der individuellen Lebensqualitätserfassung sind die Voraussetzungen hierfür gegeben. Ebenso sind die edukativen Aspekte für die Pflegenden und Ärzte entscheidend für eine gelebte Kultur der «Integrativen Onkologie».

\section{Dr. med. Günther Spahn: \\ «Integrative Medizin - \\ Herausforderung für die \\ Versorgungsforschung"}

Integrative Medizin ist in Deutschland Versorgungsrealität in den anthroposophischen Akutkliniken (Öschelbronn, Havelhöhe, Herdecke, Filderklinik, Unterlengenhardt) sowie in spezialisierten Abteilungen weniger Universitätskliniken (z.B. Essen, Jena, Berlin, München) und in ausgewählten Allgemeinkrankenhäusern mit Fachabteilungen (z.B. Hamburg).

Im Bereich der Onkologie besteht patientenseitig ein hoher Bedarf an ganzheitlichen Behandlungspfaden, die beispielsweise in der onkologischen Regelversorgung unter anderem die Aspekte Misteltherapie, Ernährung, Bewegung, künstlerische Therapien, Psychoonkologie, Stressbewältigungsmodule, westliche und östliche Phytotherapie, Akupunktur und weitere Behandlungsverfahren einschliessen.

Krebs ist im metastasierten Stadium eine systemische Erkrankung und erfordert systemische Therapieansätze. Eine zentrale Rolle nimmt dabei die Chemotherapie ein, die seit den 1950er-Jahren entwickelt wurde. Trotz grosser Erfolge in der Eindämmung der Toxizität einer zytostatischen Behandlung leiden viele Menschen an den kurz- und mittelfristigen Folgen der systemischen Chemothe- rapie. Daher hat sich allmählich eine Supportivmedizin entwickelt, die sich der Linderung von Nebenwirkungen widmet und zum vierten Instrumentarium in der konventionellen Krebsmedizin (Chirurgie, Chemotherapie, Strahlentherapie) wurde. In der Supportivmedizin spielen komplementärmedizinische Ansätze in der Onkologie eine wesentliche Rolle.

Eine an der Lebensqualität orientierte ganzheitliche Sicht ist auch in der Palliativmedizin vorhanden, die in Deutschland seit 20 Jahren zunächst zögerlich, in den letzten $5 \mathrm{Jah}$ ren jedoch mit hoher Dynamik eingeführt wurde. Mit einer Vielzahl von Palliativabteilungen, Hospizen, SAPV(Spezialisierte Ambulante Palliativversorgung)-Netzwerken und der Verankerung von palliativmedizinischen Inhalten im Medizinstudium hat die Palliativmedizin in Deutschland inzwischen einen festen Stellenwert in der medizinischen Versorgung erlangt. Auch in ihr sind komplementärmedizinische Ansätze häufig zu finden, z.B. die Behandlungsangebote mit künstlerischen Therapien, die Misteltherapie oder die Psychoonkologie.

\section{Dr. med. Helmut Lambertz:}

«Linderung therapieassoziierter Nebenwirkungen bei

Tumorpatienten»

Die Chemotherapie maligner Erkrankungen ist mit einem hohen Risiko unerwünschter Nebenwirkungen assoziiert. Daher kommt der Auswahl für die Behandlung geeigneter Patienten eine hohe Bedeutung zu, insbesondere unter dem Gesichtspunkt einer immer älter werdenden Bevölkerung.

Höheres Lebensalter geht häufig einher mit vermehrter Komorbidität, Polymedikation und funktionellen Einschränkungen. Dies führt zu einem verstärkten Auftreten von Nebenwirkungen, sodass die Dosierung und Überwachung der Chemotherapie entsprechend angepasst werden müssen. Hierzu ist ein geriatrisches

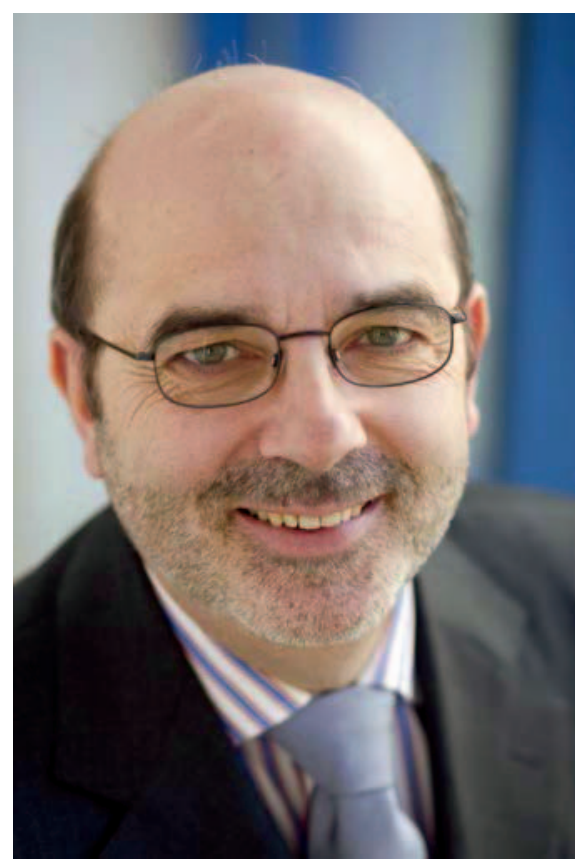

Abb. 2. Dr. med. Helmut Lambertz.

Assessment älterer Patienten unerlässlich.

Die Befürchtungen der Patienten vor Beginn einer Chemotherapie betreffen in erster Linie Fatigue und Übelkeit. Fatigue bezeichnet ein multifaktorielles Syndrom, das einer medikamentösen Therapie nur schwer zugänglich ist und in erster Linie durch körperliche Bewegung gebessert werden kann. Demgegenüber können Übelkeit und Erbrechen in den meisten Fällen durch eine konsequente antiemetogene Prophylaxe vermieden werden.

Dennoch kann es bei einigen Patienten trotz konsequenter Prophylaxe zu Übelkeit und/oder Erbrechen kommen; in diesen Fällen ist ein Breakthrough-Konzept (etwa mit Dexamethason und einem NK1-Antagonisten) hilfreich. Im Endstadium nahezu aller Krebserkrankungen kommt es zur Tumorkachexie. Dabei handelt es sich um eine komplexe Immunreaktion des Körpers auf den wachsenden Tumor.

Es gibt nur wenige gut untersuchte medikamentöse Therapieansätze, die über eine Ernährungsberatung hinaus hilfreich sein können. Eine wesent- 
liche, häufig unterschätzte Nebenwirkung stellt die Kardiotoxizität dar, die vor allem für langzeitüberlebende Krebspatienten eine erhebliche Einschränkung der Lebensqualität zur Folge hat. Daher ist ein entsprechendes Monitoring, etwa von Puls und Blutdruck, ebenso wichtig wie eine konsequente Behandlung der betroffenen Patienten.

\section{Dr. med. Matthias Rostock:}

«Klimakterisches Syndrom beim

Mammakarzinom - komplementäre

Therapieoptionen»

Die endokrine Therapie des Mammakarzinoms ist sehr effektiv, aber sie führt bei vielen Patientinnen $\mathrm{zu}$ Nebenwirkungen mit erheblichem Leidensdruck, insbesondere $\mathrm{zu}$ klimakterischen Beschwerden wie Hitzewallungen, Ängsten, Depressionen, Libidoverlust und trockenen Schleimhäuten sowie muskuloskelettalen Schmerzen. Zahlreiche Übersichtsarbeiten zeigen günstige Therapieeffekte auf, einige dieser Beschwerden durch eine begleitende Behandlung mit selektiven Serotonin-Wiederaufnahmehemmern, Gabapentin oder Clonidin zu lindern, aber ein nicht geringer Anteil der Patientinnen lehnt eine derartige Zusatzbehandlung wegen zusätzlicher Begleitbeschwerden

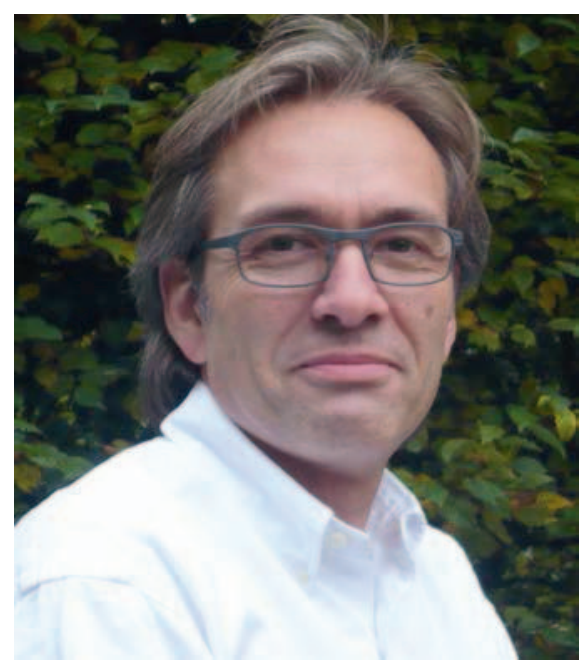

Abb. 3. Dr. med. Matthias Rostock. oder auch aus grundsätzlichen Erwägungen ab. Die Nachfrage nach komplementären Therapiemöglichkeiten ist infolgedessen gross.

Mehrere klinische Studien mit isolierten Soja-Isoflavonen haben positive Ergebnisse in der Behandlung von Beschwerden in der physiologischen Menopause ergeben, aber drei randomisierte kontrollierte Studien, die mit Brustkrebspatientinnen durchgeführt worden waren, hatten für verschiedene untersuchte Dosierungen keine Überlegenheit gegenüber Placebo ergeben.

Drei von fünf klinischen Studien mit Traubensilberkerzenextrakt zeigten eine Reduktion von Hitzewallungen; in einer vierten Studie war kein Effekt auf die Frequenz von Hitzewallungen, aber auf die Beschwerden durch Schwitzen beobachtet worden, und in der fünften Studie bestand diesbezüglich kein Unterschied zwischen Verum und Placebo.

In einer Phase-II-Studie war unter der täglichen Einnahme von $40 \mathrm{~g}$ Leinsamenschrot (400 mg Lignane) eine 50\%ige Reduktion von Hitzewallungen beobachtet worden, aber eine darauf aufbauende Phase-IIIStudie, in der der Effekt eines Leinsamenschrot-haltigen (7 g) Getreideriegels (410 mg Lignane) gegenüber einem Getreideriegel ohne Leinsamenschrot und ohne Lignane untersucht worden war, ergab keinen signifikanten Unterschied zwischen beiden Gruppen.

Prof. Dr. med. Christoph Konrad: «Behandlung von Tumorschmerzen» Opiate sind weit mehr als reine Analgetika. Dies gilt für den Akutschmerz wie auch den Einsatz in der chronischen Anwendung. Weit über 200000 Arbeiten finden sich mittlerweile zum Thema Opiate in der Datenbank Pubmed. Outcome-Forschung nimmt an Bedeutung im Bereich der klinisch orientierten Forschung zu. Im Folgenden werden einige neue Aspekte der Opiattherapie im Bereich Onkologie und Kardiologie vorgestellt.

\section{Onkologie}

Behandelte Karzinome treten beim älteren Patienten auch nach Behandlung in einem relevanten Mass wieder auf und tragen zu einer hohen Mortalität bei (52\%). Erklärt wird dies durch den Tumor selbst, aber auch durch Tumoraussaat beispielsweise bei einer onkologischen Operation. Darüber hinaus unterdrücken Operation und Anästhesie die Immunabwehr (natural killer cells). Die Gabe von Opiaten zur Analgesie oder Anästhesie kann nun folgende onkologische Mechanismen unterstützen: Tumorproliferation, Tumormigration, Angiogenese, Bildung von Proteasen und Extravasation. Dies kann die Tumorprogression oder das Wiederauftreten unterstützen. Neuere Daten weisen nun darauf hin, dass die regionale oder systemische Gabe von Lokalanästhesika solche Mechanismen inhibiert. Dies wurde für einige Tumorentitäten bereits nachgewiesen (Mamma-CA, Prostata-CA).

\section{Kardiologie}

Unter dem Begriff «Ischemic Preconditioning» versteht man einen Mechanismus, der durch eine ischämische Phase eines entfernten Organsystems einen protektiven Effekt beispielsweise auf das Myokard aufweist. Die eigentliche Molekularbiologie dahinter ist noch unklar; bekannt ist allerdings, dass die intrazelluläre Kalziumausschüttung reduziert wird. Dies kann mit der Gabe eines reinen $\mu$-Agonisten wie Morphin erreicht werden. Beschrieben sind diese protektiven Effekte für Myokard und auch Gehirn.

\section{Fazit}

Beide Beispiele zeigen, dass mit Opiaten extraanalgetische Effekte erzielt werden können. In der Behandlung kann dies durchaus klinisch re- 
levant werden, auch wenn es weiterhin gilt, dass im Bereich Onkologie die Gabe von Opiaten ein Pfeiler der Schmerztherapie ist. Dennoch gilt: «Spare Opiate in der Onkologie, gebe sie bei Myokardischämie.»

\section{Helmut Knorr:}

«Ganzheitliche Ansätze

in der Palliativtherapie»

Ganzheitlichkeit ist keine Frage der Methode, sondern eine Frage der Haltung gegenüber dem Patienten und seiner spezifischen Problemstellung. Sind wir Therapeuten bereit, ihn in seiner physischen, psychischen, spirituellen und sozialen Dimension wahrzunehmen und seinen Bedürfnissen auf diesen Ebenen gerecht zu werden?

Palliative Care ist in diesem Sinne ein Konzept in der letzten Lebensphase, das neben einer ganzheitlichen Erfassung der Problemstellung auch einen ganzheitlichen Therapieansatz bietet und methodisch zunehmend Elemente aus dem Bereich der Komplementärmedizin integriert.

\section{Dr. med. Boris Müller-Hübenthal: \\ «Immunmodulation mittels \\ Mistelpräparaten»}

Moderne multimodale Therapiekonzepte haben in den letzten Jahren dazu beigetragen, die Therapieergebnisse bei vielen Krebserkrankungen deutlich zu verbessern. In onkologischen Schwerpunktkliniken werden Chirurgie, Strahlentherapie und Chemotherapie kombiniert mit Psychoonkologie, Physiotherapie und Ernährungsmedizin. Zudem spricht eine zunehmende Evidenz für den ergänzenden Einsatz komplementärer Therapieverfahren.

Im Rahmen der Integrativen Onkologie wird die spezifische Krebstherapie mit supportiven und komplementärmedizinischen Therapieverfahren verbunden. Die Gruppe der anthroposophischen Mistelpräparate gehört zu den am häufigsten eingesetzten komplementären Tumortherapien in Mitteleuropa. Ihr Einsatz erfolgt unter der Vorstellung einer direkten antitumoralen Wirkung zur Verbesserung der Lebensqualität und bei gleichzeitiger Abschwächung therapieassoziierter Toxizität.

Mistel enthält Lektine (Lektin I, II und III; hochmolekulare Polypeptide), lösliche Triterpene (insbesondere Oleanol-Säure), Terpenoide und Viscotoxine A2, A3 und B (niedermolekulare Polypeptide). Die Misteltherapie führt zu einer unspezifischen Stimulation des Immunsystems und zur beschleunigten Knochenmarksregeneration nach zytostatischer Therapie.

Die grösste Bedeutung der Misteltherapie liegt in der verbesserten Lebensqualität. Auf der physischen Ebene werden unter anderem Schmerzen und das Tumor-Fatigue-Syndrom reduziert und der Appetit verbessert. Auf der seelischen Ebene können depressive Symptome gelindert und der Antrieb gesteigert werden.

Damit werden Voraussetzungen geschaffen, die eine positive Lebens-

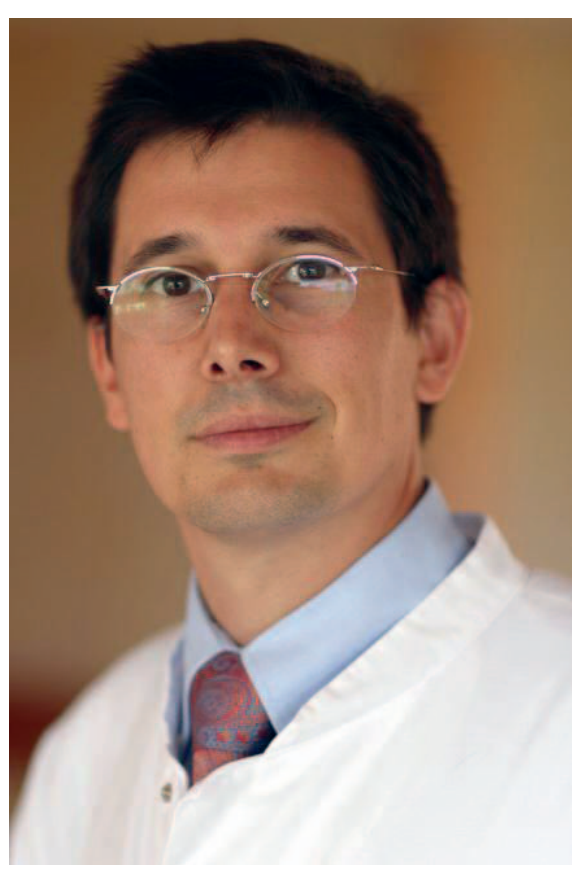

Abb. 4. Dr. med. Boris Müller-Hübenthal. gestaltung und Entwicklung mit der Erkrankung ermöglichen, auch wenn diese nicht mehr heilbar sein sollte.

\section{Dr. med. Axel von Manitius:}

«Phytotherapie beim Prostatakrebs» Aktuelle Studien belegen zunehmend den Nutzen einer phytotherapeutischen Begleitbehandlung beim Prostatakarzinom. Ein Einsatz im Rahmen der Active Surveillance, des Watchful Waitings und begleitend zur Hormontherapie scheint sinnvoll. Insbesondere Granatapfelpolyphenole und Tectorigenin aus Belamcanda chinensis liefern vielversprechende Ergebnisse in Untersuchungen an Zellkulturen, in Tierexperimenten und auch in Untersuchungen am Menschen.

Die Wirksamkeit synthetischen Östrogens auf das Prostatakarzinom ist hinlänglich bekannt und belegt. Sein Einsatz ist seit der Einführung derLHRH-Analoga und Antiandrogene allerdings aufgrund des ungünstigen Nebenwirkungspotenzials (thromboembolische Komplikationen) unüblich geworden. Untersuchungen und Erfahrungen mit östrogenhaltigen Mischpräparaten (Diethylstilbestrol + Phytoöstrogene + Flavonoide) zeigen eine gute Wirksamkeit bei der Bekämpfung des Prostatakarzinoms bei insgesamt geringerer Komplikationsrate. Wir setzen daher in der Aeskulap-Klinik zur Behandlung des nicht kurativ behandelbaren, hormonrefraktären Prostatakarzinoms eine entsprechende Magistralrezeptur (Sitosterol comp.) ein. Die seit 2010 gewonnenen Daten zur Wirksamkeit und Sicherheit von Sitosterol comp. wurden vorgestellt. Hierbei konnten in einer retrospektiven Untersuchung 27 Probanden mit einer Mindestbehandlungsdauer von 3 Monaten eingeschlossen werden. Bei $40 \%$ wurde eine partielle Remission, bei $56 \%$ eine «stable disease» und bei nur 4\% eine Progression bei insgesamt niedriger Nebenwirkungsrate festgestellt. 


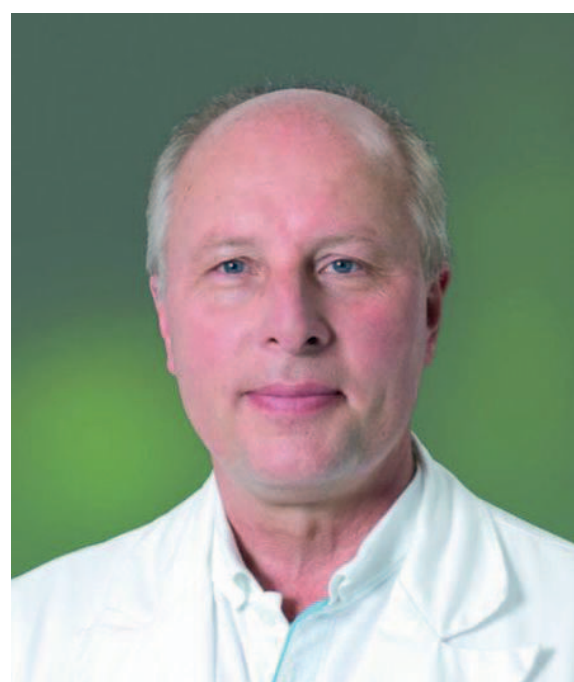

Abb. 5. Dr. med. Friedrich Migeod.

Dr. med. Friedrich Migeod: «Lokoregionale Radiofrequenztiefenhyperthermie, hypertherme Perfusionschemotherapie»

Hyperthermie ist die über der Körpertemperatur liegende Erwärmung von einzelnen Organen oder umschriebenen Körperteilen, meist eingesetzt bei malignen Tumoren mit dem Ziel einer irreversiblen Zellschädigung. Hierzu werden Techniken, die Radiofrequenzhyperthermie mit resistiven (Frequenz 0,5-1,0 MHz) oder kapazitiv angekoppelten Elektroden (8-27 MHz) eingesetzt - ebenso die Mikrowellenhyperthermie mit radiativ wirksamen koaxialen Monopoloder Dipolantennen (Frequenz 434$2450 \mathrm{MHz}$ ). Sehr wirksam, aber in der Handhabung kompliziert, ist in diesem Bereich auch die Ultraschallhyperthermie mit aklistisch wirksamen Piezokristall-Applikatoren, bestehend aus mehreren Segmenten (Frequenz 0,5-5 MHz). Im Gegensatz dazu steht die Thermotherapie, die an der Körperoberfläche (Infrarothyperther- mie) und mit konduktiv wirksamen Heisswasserapplikatoren (HOT-Source-Hyperthermie) eingesetzt werden kann. Im Körperinneren kommen die Thermoablation mit durch Gleichstrom beheizten Nadeln, die Laserhyperthermie mit verschiedenen Laser- und Glasfasersystemen (NDYAG-Laser (1064 nm) oder Diodenlaser (800-1000 nm)) und auch sogenannte Thermoseeds entsprechend ferromagnetischen Implantaten in der Prostata und umschriebenen Hirntumoren zur Anwendung.

Die Mikrowellenhyperthermie zerstört die Tumorzelle durch eine hohe absolute Temperatur, die sich sowohl intra- und extrazellulär als auch im benignen und malignen Gewebe findet und somit eine sehr präzise Lokalisation mit Einbringung einer Temperatursonde erfordert. Während durch die Mikrowellenhyperthermie eine «direkte» Zerstörung des Tumorgebiets möglich ist und das Erzielen einer effektiven Temperatur von über $40{ }^{\circ} \mathrm{C}$ unverzichtbar ist, erzeugt die Hyperthermie mit kapazitiv gekoppelten Elektroden, meist im Frequenzbereich von $13,56 \mathrm{MHz}$, vielfältige Effekte, wie Proteindenaturierung, Hypoxie, Hypoglykämie, Antiangiogenese, Hemmung von DNA-RepairMechanismen und Induktion von Hitzeschockproteinen mit Erzeugung einer regionalen Immunantwort - vor allem der zytotoxischen Zellen.

\section{Dr. med. Christoph Kittel:}

"Lokoregionale Tiefenhyperthermie eigene Erfahrungen»

In der Aeskulap-Klinik wird die lokoregionale Tiefenhyperthermie

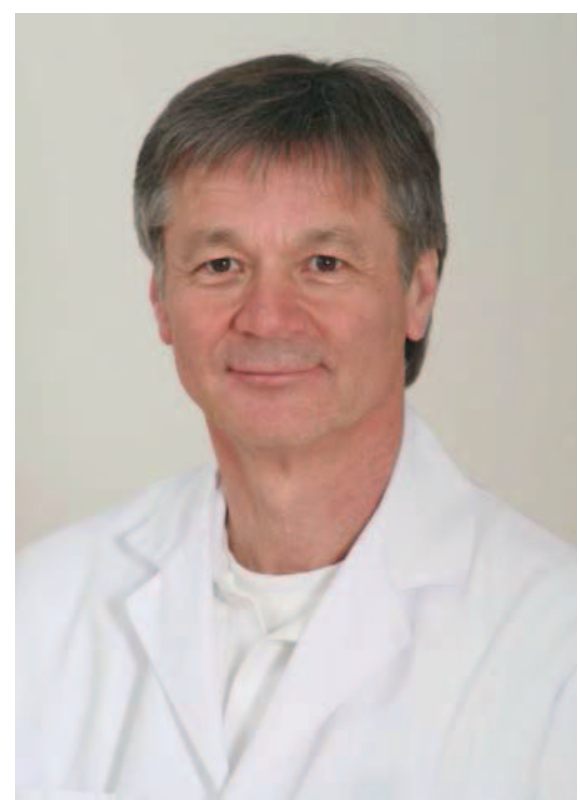

Abb. 6. Dr. med. Christoph Kittel.

seit 3,5 Jahren mit dem Celcius-TCSGerät der Firma Celsius42+ durchgeführt. Zwischenzeitlich wurden ca. 150 Patienten mit unterschiedlichen Tumorentitäten meist in Begleitung zur Chemo-, Radio- oder Antikörpertherapie behandelt.

Langzeit-Hyperthermieanwendungen begleitend zur Chemoerhaltungstherapie lassen Tendenzen ausserordentlich stabiler Verläufe erkennen. Anhand von Patientenbeispielen werden solche Langzeitverläufe geschildert.

Auffallend ist die Häufung von Langzeit-Hyperthermiebehandlungen bei bindegewebigen und weichgewebigen Malignomen, wie dem malignen Mesotheliom und Weichteilsarkom. Im Vortrag wurden Beispiele von Behandlungsverläufen genannt, die einen Einfluss der Langzeit-Hyperthermie auf den Behandlungsverlauf nahelegen. 